\title{
Biochemistry and Neuroscience: The Twain Need to Meet
}

\author{
Mary B. Kennedy \\ Division of Biology and Biochemical Engineering \\ Mail Code 216-76, California Institute of Technology \\ Pasadena, CA 91125 \\ kennedym@its.caltech.edu
}


Bruce Alberts and others introduced the concept of "molecular machines" about two decades ago to describe large assemblies of biomolecules that are specialized to perform particular cellular functions [1]. Much of modern cell biology involves the study of such molecular machines. The goal is to understand how they execute and coordinate the multitude of cell functions required for life. Active areas of study include the intricate structures that form transcriptional complexes [2], the nuclear pores that control movement of macromolecules between nucleus and cytoplasm [3], transient vesicle structures that transport molecular machines to distal parts of the cell [4], and the emerging study of membrane-less compartments, such as the nucleolus [ $\left.5^{*}\right]$. Neurons contain unique molecular machines, but their study has often lagged behind those that are common to most cell types.

The word "synapse" used to be defined as the gap between the presynaptic terminal and the postsynaptic site. However, for those of us who study synaptic function, the word has come to mean the combination of the presynaptic bouton where transmitter is released, the synaptic cleft, and the plaque of postsynaptic neurotransmitter receptors and associated protein structures that regulate their assembly. Excitatory synapses in the mammalian brain comprise a particularly large, complicated set of protein machines specialized to process information about the environment in real time and then store it by adjusting the strengths of synapses that connect neurons when they are activated together by an environmental event.

Mechanisms of transmitter release by exocytosis from synaptic vesicles are closely related to fundamental mechanisms of exocytosis in many cells [e.g. 6]. As a result, synaptic vesicles isolated from the brain have often served as a convenient and abundant source of biochemical material to study the exocytotic machinery. Biochemical studies of individual purified vesicle proteins, structural studies at the electron microscopic and atomic level, and sophisticated microphysiological measurements with electrodes have all contributed to our present understanding of the interactions of vesicle proteins with the "SNARE" machinery that ultimately brings about release of neurotransmitter into the synaptic cleft [7**].

In contrast, study of molecular mechanisms in the postsynaptic spines of excitatory synapses has been more fraught. Just one year after Alberts published his article on molecular machines in the cell, Sanes and Lichtman published a review in Nature Neuroscience with the improbable title, "Can Molecules Explain LTP?" [8]. To understand the gulf between the emerging biochemistry of the cell at the turn of the century, and the molecular sophistication of the neuroscience field, simply imagine the response if a prominent cell biologist had published a review entitled, "Can molecules explain the cell cycle?" or "Can molecules explain gene expression?". In 1998, the notion that molecular explanations of complex cellular functions were in sight had permeated the field of cell biology. In contrast, neuroscientists, most of whom are trained fundamentally in electrophysiology or biophysics, were all too ready to give up, for the time being, on molecular explanations of neuronal function. 
Unfortunately, cell biologists don't generally view the molecular machinery that forms and remodels the postsynaptic plaque of receptors in spines as representative of universal cellular processes. They have generally viewed these neuronal structures as more complex and heterogenous than machinery that regulates receptors in other tissues and thus less amenable to biochemical study. This situation is reflected in the composition of textbooks covering cell biology. Many of them mention synaptic vesicle proteins when discussing exocytosis. However, chapters on integrated intercellular signaling use the immune system as an example, and avoid the complexities of postsynaptic signaling [e.g. 9]. Conversely, neuroscience textbooks generally cover molecular mechanisms with simplified cartoons [e.g. 10,11].

A relatively small cohort of intrepid researchers, usually located in molecular neuroscience laboratories, have tackled the molecular mechanisms of formation and pruning of synapses, and the modulatory mechanisms that tune the strengths of synapses ("synaptic plasticity"). Through their efforts, usually carried out in isolation from laboratories focused on cell biology and biochemistry, most of the key individual proteins involved in postsynaptic signaling and modulation have been identified. However, our understanding of how these proteins work together to provide the subtle, yet vital tuning of synaptic machinery necessary to support stable brain functions lags behind, and is difficult to fund. A deep understanding of the properties and behavior of individual proteins and their interactions as they assemble into protein machinery is as crucial for a full understanding of neuroscience as it is for all branches of cell biology. In this review, I will discuss two examples from the recent literature of what I believe to be oversimplified interpretation of studies of synaptic plasticity in living neuronal preparations, and the deeper understanding that arises from taking into account the biochemical properties of individual proteins.

\section{The role of PKM $\zeta$ in maintenance of late-phase LTP}

It has been customary to divide mechanisms of long term potentiation of excitatory synapses (LTP) into two broad phases (see Fig. 1C). The induction phase refers to the period during which synaptic activity initiates biochemical changes that ultimately result in stable potentiation, and is usually considered to include the first 10 to 20 minutes after an inducing stimulus. The maintenance phase refers to the period after induction during which the synapse has reached a new level of strength that persists for an indefinite period. It is usually considered to begin $\sim 20$ minutes after the inducing stimulus. The term "late-LTP" refers to the period from $\sim 1$ hour to as long as an experiment lasts, which for experiments carried out on hippocampal slices, can be up to 9 hours.

A great deal of attention has been focussed on the mechanisms of induction. We know that the ultimate strength of potentiation and its endurance depend on the nature of the inducing stimulus. Thus investigators have sought to explain how various synaptic stimuli alter synaptic biochemistry in the few minutes after the stimulus. At this time, all agree that the critical early event is activation of NMDA-type glutamate receptors that gate a large flux of $\mathrm{Ca}^{2+}$ into the postsynaptic cytosol [12]. It is also agreed that activation of 
phosphorylation events by the abundant postsynaptic protein kinase, $\mathrm{Ca}^{2+} /$ calmodulindependent protein kinase II (CaMKII) initiates a cascade of biochemical changes that ultimately remodel the synapse to contain more AMPA-type glutamate receptors and larger pre- and postsynaptic specializations [13]. What is less clear, and more controversial, are the precise biochemical changes that cause a potentiated synapse to maintain its increased strength essentially indefinitely, an indurance that is necessary for formation of long-lasting memory [14].

Recently, a major clue about the mechanisms of maintenance of LTP fell firmly into place: Increased synthesis of one particular stable isoform of the atypical protein kinase $\mathrm{C} \zeta$ (PKC $\zeta)$, termed PKM $\zeta$, is necessary for fully maintained LTP, and can stably increase synaptic strength when its concentration is increased in a postsynaptic spine.

The first work leading to this now well established conclusion was presented in a series of carefully executed and beautifully written papers by Todd Sacktor's lab from 1993 to 2004. It began with a paper in which they showed that a constitutively active fragment of $\mathrm{PKC} \zeta$, referred to as $\mathrm{PKM} \zeta$, is upregulated in hippocampal slices during the maintenance phase of LTP [15]. At that time, PKM $\zeta$ was thought to be generated by proteolysis of PKC $\zeta$ by the $\mathrm{Ca}^{2+}$-dependent protease calpain. However, the Sacktor lab soon found that the increased formation of PKM $\zeta$ following induction of LTP was dependent on protein synthesis [16], and that, contrary to previous assumptions, $\mathrm{PKM} \zeta$ is a true alternative gene product of the $\mathrm{PKC} \zeta$ gene [17]. The mRNA encoding $\mathrm{PKM} \zeta$ is transcribed from an alternative promoter of the gene, and $\mathrm{PKM} \zeta$ is translated de novo from that mRNA. The $\mathrm{PKM} \zeta \mathrm{mRNA}$ is rapidly transported from the nucleus into dendrites [18] and $\mathrm{PKM} \zeta$ is the major form of the kinase expressed from the $\mathrm{PKC} \zeta$ gene in hippocampus and neocortex [17]. Much of this foundational work involved traditional biochemical experiments carried out in the Sacktor lab.

To examine the role of PKM $\zeta$ in maintenance of LTP and the persistence of memory, the Sacktor lab used a known pharmacological inhibitor of $\mathrm{PKM} \zeta$, chelerythrine, and developed a second, myristoylated $\zeta$-pseudosubstrate (later named ZIP) based on the sequence of the inhibitory domain of $\mathrm{PKC} \zeta$. A key set of electrophysiological experiments [19] demonstrated that specific inhibition of PKM $\zeta$ one hour after induction of LTP in hippocampal slices by either chelerythrine or the ZIP inhibitor reversed LTP. Furthermore, introduction of recombinantly expressed $\mathrm{PKM} \zeta$ into a pyramidal neuron through a patch electrode increased the evoked excitatory postsynaptic currents as much as $100 \%$ in 10 min. This set of experiments appeared to establish the unique importance of PKM $\zeta$ for maintaining the later phases of LTP. Several studies have since shown that introduction of ZIP peptide by infusion into various regions of the mouse brain can reverse learning of several behaviors as long as 3 months after the learning occurred [20].

Nonetheless, a fly appeared in the ointment in 2013 in the form of two papers published in the journal "Nature" showing that mice with targeted deletion of the PKC $\zeta$ gene, a mutation that also eliminates expression of $\mathrm{PKM} \zeta$, showed normal synaptic plasticity in the hippocampus [21] and normal long-term learning of several behavioral tasks [22]. 
Furthermore, the ZIP peptide inhibited LTP and could reverse memory of learned tasks when infused into the brains of trained mutant mice missing PKM $\zeta$. These surprising experiments seemed to indicate that another, as yet unknown, protein, also inhibited by ZIP, was the true mediator of maintenance of LTP and memory formation. One of the papers [21] mentioned the possibility that an atypical PKC that is closely related to PKM $\zeta$, $\mathrm{PKC} \lambda / \mathrm{l}$, might also be inhibited by ZIP and might be "compensating" for PKM $\zeta$. However, the investigators cast doubt on this possibility after failing to find increased expression of $\mathrm{PKC} \lambda / \mathrm{l}$ two hours after induction of LTP in the mutant mice.

Drawing upon their deep understanding of the biochemistry of atypical PKC's, the Sacktor group next devised a series of carefully controlled experiments [23**] that established the following key facts: 1 . PKC $\lambda / \mathrm{l}$ is indeed inhibited by ZIP, although at a slightly higher concentration of ZIP than required for inhibition of PKM $\zeta ; 2$. Transient knockdown of PKM $\zeta$ with antisense RNA inhibits maintenance of LTP in hippocampal slices from wild type mice, but does not inhibit it in slices from PKM $\zeta$ null mice; 3. The amount of $\mathrm{PKC} \lambda / \mathrm{l}$ is transiently increased after tetanization of slices from wild type mice, but the increase becomes persistent in PKM $\zeta$ null mice and lasts for at least 3 hours after the tetanus (Fig. 1A); 4. A newly established specific inhibitor of $\mathrm{PKC} \lambda / \mathrm{l}$, termed ICAP [24,25], reverses late-LTP maintenance in PKM $\zeta$ null mice, but not in wild type mice (Fig. 1B, C); and 5. Similar differences between wild type and PKM $\zeta$ null mice are found for inhibition of behavioral learning.

In addition to re-establishing that $\mathrm{PKM} \zeta$ is indeed a primary regulator of maintenance of late-LTP in wild type mice [26*], these experiments uncovered a subtle redundancy in the maintenance mechanism. In wild type animals, new synthesis of PKM $\zeta$ after LTP-inducing stimuli produces a down-regulation of the synthesis of $\mathrm{PKC} \lambda / \mathrm{l}$. However, when $\mathrm{PKM} \zeta$ is deleted, the down-regulation doesn't occur and $\mathrm{PKC} \lambda / \mathrm{l}$ remains elevated for a longer period. The Sacktor group went on to show that the compensation by $\mathrm{PKC} \lambda / \mathrm{l}$ in $\mathrm{PKM} \zeta$ null mice isn't perfect [23], as might be expected from the principles of evolution. When given increasingly demanding cognitive tests, the PKM $\zeta$ null mice began to perform measurably less well than wild type mice.

In this example, deep knowledge of the biochemical characteristics of the enzymes being studied (PKM $\zeta$ and $\mathrm{PKC} \lambda / \mathrm{l}$ ), and the mechanisms by which they are regulated, was necessary to fully unravel the primary importance of PKM $\zeta$ in the wild type and the subtly intertwined roles of the two enzymes in maintenance of LTP and memory. Without it, the two genetic studies that found PKM $\zeta$ to be unnecessary for maintenance of late LTP might have stood as the last word and the field would have been set back.

\section{The role of synGAP in induction of LTP}

Many electrophysiologists say that the immediate results and instant interaction with a neuronal preparation that occurs during their experiments is the genesis of their passion for neuroscience. Perhaps for this reason, neuroscience students are often taught that "real time" imaging of heterologously expressed fluorescent proteins in vivo coupled with electrophysiology (sometimes called "molecular replacement experiments") is the gold stan- 
dard for mechanistic studies. The erroneous notion that these methods are "more physiological" than studies of purified proteins ignores the disruption of intracellular biochemistry that is created by over-expression (or under-expression) of fluorescently labeled heterologous proteins in cells. Principles of physical biochemistry tell us that both the timing (kinetics) and the binding affinities that normally govern interactions between intracellular molecules are altered by over- and under-expression, as well as by modification of proteins with hydrophobic fluorescent moieties. When appropriately controlled, these methods are certainly useful to test ideas about the behavior of well-understood individual proteins in vivo, but the introduction of altered molecules always perturbs the system. Therefore, these methods are not adequate by themselves to unravel complex molecular mechanisms or reveal the fine-tuned workings of synaptic molecular machinery. An accurate interpretation of such experiments requires an understanding of the intrinsic properties of the proteins, including enzymatic rates and binding constants. These properties can only be adequately understood by studying purified proteins in vitro.

I illustrate this assertion with recent studies of the roles of the protein synGAP in the early stages of induction of LTP. SynGAP is an abundant cytosolic regulatory enzyme that localizes specifically to the postsynaptic spine and nearby dendrite. It binds to the scaffold structure underlying the postsynaptic membrane, known as the postsynaptic density (PSD) [13]. SynGAP contains a Ras GTPase activating domain that interacts with activated Ras protein to accelerate the rate of hydrolysis of its bound GTP molecule, which inactivates Ras $[27,28]$. A C2 domain adjacent to the RasGAP domain confers additional specificity for acceleration of inactivation of Rap [29]. SynGAP is held near the postsynaptic membrane, in part, because one of its alternatively spliced variants, termed synGAP- $\alpha 1$, has a PDZ domain ligand that binds to the PDZ domains of PSD-95 [see 30]. PSD-95 is a major PSD scaffold protein and binds many postsynaptic receptors and signaling proteins via its three PDZ domains [13].

Two recent imaging studies showed that activation of $\mathrm{Ca}^{2+} /$ calmodulin-dependent protein kinase II (CaMKII) by synaptic NMDA-type glutamate receptors (NMDARs) causes synGAP to move away from the core of the PSD. One study (Fig. 2) showed by electron microscopy that two major isoforms of synGAP, synGAP- $\alpha 1$, which contains the PDZ ligand, and synGAP- $\alpha 2$ which does not, move away from the synaptic membrane after phosphorylation and appear to associate with a cloud of material that underlies and surrounds the PSD proper [31]. A second study showed that fluorescent synGAP- $\alpha 1$, introduced into neurons by "molecular replacement", disperses away from the synaptic junction when NMDARs on individual spines are activated by "chemLTP" [32*]. It appears to move into the adjacent shaft where it is diluted. Araki et al. [32] theorized that the dispersal of synGAP removes a negative influence on the activation of Ras at the synapse. Indeed, they used a fluorescent FRET indicator to show, as have others [33], that the amount of active Ras in a spine increases upon activation of NMDARs and subsequent movement of synGAP out of the spine. They concluded that movement of synGAP away from the PSD facilitates activation of Ras, which is necessary for increases in spine size and addition of new AMPA-type glutamate receptors (AMPARs) to the spine membrane [32]. 
In contrast, two recent biochemical studies from my lab indicate that the mechanism underlying addition of new AMPARs is both more subtle and more complex than proposed by Araki et al. It has been known for some time that SynGAP accelerates inactivation of Rap as well as Ras [29,34]. Thus, movement of synGAP out of the spine would be expected to activate Rap, as well as Ras. Expression of active Rap in neurons accelerates endocytosis of AMPARs; whereas, expression of active Ras has the opposite effect, accelerating exocytosis of AMPARs [35]. The fold stimulation of Rap inactivation by synGAP is considerably higher than its stimulation of inactivation of Ras [36*]. This means that simple loss of synGAP from the spine would be expected to cause a greater increase in active Rap in the spine and a loss of surface AMPARs, in contrast to the model proposed by Araki et al.. Indeed, we found that phosphorylation of synGAP by CaMKII doubles the rate of synGAP's inactivation of Rap [36]. Thus, one of the same phosphorylation events that promotes detachment of synGAP from the PSD, also causes its rate of inactivation of Rap to increase, not decrease. This would result in a rapid reduction of active Rap, and a concomitant reduction of endocytosis, along the peripheral spine and dendritic membrane, as synGAP disperses away from the PSD. Our results suggest that, rather than eliminating the effect of synGAP on Ras and Rap activity, the rheostat-like shifting of the enzymatic specificity of synGAP by phosphorylation would shift the balance toward exocytosis of receptor at the extrasynaptic membrane, as synGAP moves away from the PSD. Interestingly, phosphorylation of another site on synGAP by the homeostatic enzyme Cdk5, increases the rate of inactivation of Ras, without altering the rate of inactivation of Rap, turning the rheostat in the opposite direction, presumably leading to reduction of the number of surface receptors [36].

In a second biochemical study, we uncovered a function of synGAP unrelated to its GAP activity [37**]. We found that phosphorylation of synGAP by CaMKII at several sites progressively reduces the affinity of synGAP binding to all three PDZ domains of PSD-95. This effect involves phosphorylation of more sites than identified in [32] and has more consequences than simple movement of synGAP out of the PSD. Because synGAP is remarkably abundant in the PSD, nearly as abundant as PSD-95 itself, we theorized that it might serve as a placeholder to regulate the number of PDZ domain "slots" on the scaffold available for binding AMPARs or other proteins. To test this idea, we measured the relative abundance of several PSD proteins in isolated PSD fractions from mouse brains, normalizing all of the measurements to the corresponding amounts of PSD-95. We compared the abundance of proteins in PSDs from wild type mice to those from mice with a deletion of one copy of the synGAP gene, which halves the amount of synGAP in the brain [38]. As expected, mice with the synGAP deletion have less synGAP per PSD-95 molecule (Fig. 3A). Somewhat to our surprise, the experiment also revealed that PSDs from mice with the synGAP deletion have a significantly larger amount of AMPA receptor chaperone proteins (TARPs [39] and LRRTMs [40]) that help anchor AMPA receptors into the PSD by binding them to PSD-95 (Fig. 3B,C). This result supports the hypothesis that synGAP acts as a placeholder by occupying PDZ domains "slots" on PSD-95. The effects of phosphorylation 
on affinity of synGAP for PDZ domains of PSD-95 further suggest that the placeholder function is regulated by activation of NMDARs [37].

\section{Conclusion}

Unfortunately, the relatively blunt tools of electrophysiology and imaging favored by neuroscientists do not have the resolution to parse individual biochemical steps in synaptic formation or plasticity. On the other hand, because this machinery is both complex and embedded in the heterogenous brain "neuropil", it is tricky to study by standard cell biological and biochemical methods. The erroneous notion that in vivo imaging of fluorescent proteins is "more physiological" than biochemical studies of purified proteins, and the inherent difficulty of isolating postsynaptic neuronal preparations from the brain may have discouraged investigators trained in cell biology from turning their attention to neurons and synapses. It is increasingly clear, however, that many of the most intractable mental disorders, including schizophrenia , autism, and intellectual disability, involve dysfunction of synaptic regulatory machinery. To make progress in treating these disorders, neuroscientists need to begin accepting, valuing, and funding quantitative, in vitro, biochemical studies of proteins in addition to "real-time" imaging experiments.

Acknowledgements: The author's work has been funded by the Allen and Lenabelle Davis Foundation, the Hicks Foundation for Alzheimer's Research, and by grants from the Gordon and Betty Moore Foundation (Center for Integrative Study of Cell Regulation), the National Institutes of Health, the National Science Foundation, and the Beckman Institute.

\section{References}

\section{Alberts B: The cell as a collection of protein machines: preparing the next generation of} molecular biologists. Cell 1998, 92:291-294.

2. He Y, Yan C, Fang J, Inouye C, Tjian R, Ivanov I, Nogales E: Near-atomic resolution visualization of human transcription promoter opening. Nature 2016, 533:359-365.

3. Lin DH, Stuwe T, Schilbach S, Rundlet EJ, Perriches T, Mobbs G, Fan Y, Thierbach K, Huber FM, Collins LN, et al.: Architecture of the symmetric core of the nuclear pore. Science 2016, 352:aaf1015. 
4. Dodonova SO, Diestelkoetter-Bachert P, von Appen A, Hagen WJ, Beck R, Beck M, Wieland F, Briggs JA: VESICULAR TRANSPORT. A structure of the COPI coat and the role of coat proteins in membrane vesicle assembly. Science 2015, 349:195-198.

*5. Hyman AA, Weber CA, Julicher F: Liquid-liquid phase separation in biology. Annu Rev Cell Dev Biol 2014, 30:39-58.

This review describes the molecular forces that underlie formation of membrane-less compartments such as the nucleolus, the centrosome, and, perhaps, the postsynaptic density.

6. Saheki Y, De Camilli P: Synaptic vesicle endocytosis. Cold Spring Harb Perspect Biol 2012, 4:a005645.

**7. Zhou Q, Lai Y, Bacaj T, Zhao M, Lyubimov AY, Uervirojnangkoorn M, Zeldin OB, Brewster AS, Sauter NK, Cohen AE, et al.: Architecture of the synaptotagmin-SNARE machinery for neuronal exocytosis. Nature 2015, 525:62-67.

Understanding the architecture of the protein machinery that mediates $\mathrm{Ca}^{2+}$-triggered release of transmitter required biochemical studies of the individual proteins and their complexes in vitro.

8. Sanes JR, Lichtman JW: Can molecules explain long-term potentiation? Nature Neurosci. 1999, 2:597-604.

9. Alberts B, Johnson A, Lewis J, Raff M, Roberts K, Walter P: Molecular Biology of the Cell edn sixth: Garland Press; 2014.

10. Kandel ER, Schwartz J, Jessell T, Siegelbaum SA, Hudspeth AJ: Principles of Neural Science edn 5: McGraw-Hill; 2012.

11. Bear MF, Connors BW, Paradiso MA: Neuroscience: Exploring the Brain edn 4: Wolters Kluwer; 2015.

12. Luscher C, Malenka RC: NMDA receptor-dependent long-term potentiation and long-term depression (LTP/LTD). Cold Spring Harb Perspect Biol 2012, 4.

13. Kennedy MB: Synaptic signaling in learning and memory. Cold Spring Harb Perspect Biol 2013, 8:a016824. 
14. Sacktor TC: PINing for things past. Sci Signal 2010, 3:pe9.

15. Sacktor TC, Osten P, Valsamis H, Jiang X, Naik MU, Sublette E: Persistent activation of the zeta isoform of protein kinase $\mathbf{C}$ in the maintenance of long-term potentiation. Proc Natl Acad Sci U S A 1993, 90:8342-8346.

16. Osten P, Valsamis L, Harris A, Sacktor TC: Protein synthesis-dependent formation of protein kinase Mzeta in long-term potentiation. J Neurosci 1996, 16:2444-2451.

17. Hernandez AI, Blace N, Crary JF, Serrano PA, Leitges M, Libien JM, Weinstein G, Tcherapanov A, Sacktor TC: Protein kinase M zeta synthesis from a brain mRNA encoding an independent protein kinase $\mathrm{C}$ zeta catalytic domain. Implications for the molecular mechanism of memory. J Biol Chem 2003, 278:40305-40316.

18. Muslimov IA, Nimmrich V, Hernandez AI, Tcherepanov A, Sacktor TC, Tiedge H: Dendritic transport and localization of protein kinase Mzeta mRNA: implications for molecular memory consolidation. J Biol Chem 2004, 279:52613-52622.

19. Ling DS, Benardo LS, Serrano PA, Blace N, Kelly MT, Crary JF, Sacktor TC: Protein kinase Mzeta is necessary and sufficient for LTP maintenance. Nat Neurosci 2002, 5:295-296.

20. Sacktor TC: How does PKMzeta maintain long-term memory? Nat Rev Neurosci 2011, 12:915.

21. Volk LJ, Bachman JL, Johnson R, Yu Y, Huganir RL: PKM-zeta is not required for hippocampal synaptic plasticity, learning and memory. Nature 2013, 493:420-423.

22. Lee AM, Kanter BR, Wang D, Lim JP, Zou ME, Qiu C, McMahon T, Dadgar J, FischbachWeiss SC, Messing RO: Prkcz null mice show normal learning and memory. Nature 2013, 493:416-419.

**23. Tsokas P, Hsieh C, Yao Y, Lesburgueres E, Wallace EJ, Tcherepanov A, Jothianandan D, Hartley BR, Pan L, Rivard B, et al.: Compensation for PKMzeta in long-term potentiation and spatial long-term memory in mutant mice. Elife 2016, 5. Increased synthesis of PKM $\zeta$ is necessary in wild type mice for maintenance of latephase LTP. Loss of the complex interplay between PKM $\zeta$ and PKC $\lambda / \imath$ allows PKC $\lambda / \imath$ to compensate for loss of PKM $\zeta$ during late phase LTP in a PKM $\zeta$ null mouse. 
24. Pillai P, Desai S, Patel R, Sajan M, Farese R, Ostrov D, Acevedo-Duncan M: A novel PKCiota inhibitor abrogates cell proliferation and induces apoptosis in neuroblastoma. Int J Biochem Cell Biol 2011, 43:784-794.

25. Sajan MP, Acevedo-Duncan ME, Standaert ML, Ivey RA, Lee M, Farese RV: Akt-dependent phosphorylation of hepatic FoxO1 is compartmentalized on a WD40/ProF scaffold and is selectively inhibited by aPKC in early phases of diet-induced obesity. Diabetes 2014, 63:2690-2701.

*26. Morris RG: Forget me not. Elife 2016, 5.

In this commentary on Tsokas et al. [22]. Morris suggests that, in the brain, at some very late phase of memory, potentiated synapses might become structurally stable even without the influence of enhanced synthesis of PKM

27. Kim JH, Liao D, Lau L-F, Huganir RL: SynGAP: a synaptic RasGAP that associates with the PSD-95/SAP90 protein family. Neuron 1998, 20:683-691.

28. Chen H-J, Rojas-Soto M, Oguni A, Kennedy MB: A synaptic Ras-GTPase activating protein (p135 SynGAP) inhibited by CaM Kinase II. Neuron 1998, 20:895-904.

29. Pena V, Hothorn M, Eberth A, Kaschau N, Parret A, Gremer L, Bonneau F, Ahmadian MR, Scheffzek K: The C2 domain of SynGAP is essential for stimulation of the Rap GTPase reaction. EMBO Rep. 2008, 9:350-355.

30. McMahon AC, Barnett MW, O'Leary TS, Stoney PN, Collins MO, Papadia S, Choudhary JS, Komiyama NH, Grant SG, Hardingham GE, et al.: SynGAP isoforms exert opposing effects on synaptic strength. Nat Commun 2012, 3:900.

31. Yang Y, Tao-Cheng JH, Bayer KU, Reese TS, Dosemeci A: CaMKII-mediated phosphorylation regulates distributions of SynGAP-alpha1 and -alpha2 at the postsynaptic density. PLoS One 2013, 8:e71795.

*32. Araki Y, Zeng M, Zhang M, Huganir RL: Rapid dispersion of SynGAP from synaptic spines triggers AMPA receptor insertion and spine enlargement during LTP. Neuron 2015, 85:173-189.

SynGAP is visualized dispersing away from spines in neuronal cultures activated by "chemLTP". The authors posit that this dispersion removes synGAP's inactivation of Ras from the spine, and leads to an increase in active Ras that is critical for LTP. 
33. Yasuda R, Harvey CD, Zhong H, Sobczyk A, van Aelst L, Svoboda K: Supersensitive Ras activation in dendrites and spines revealed by two-photon fluorescence lifetime imaging. Nat Neurosci 2006, 9:283-291.

34. Krapivinsky G, Medina I, Krapivinsky L, Gapon S, Clapham DE: SynGAP-MUPP1-CaMKII synaptic complexes regulate 338 MAP kinase activity and NMDA receptor-dependent synaptic AMPA receptor potentiation. Neuron 2004, 43:563-574.

35. Zhu JJ, Qin Y, Zhao M, Van Aelst L, Malinow R: Ras and Rap control AMPA receptor trafficking during synaptic plasticity. Cell 2002, 110:443-455.

*36. Walkup IV WG, Washburn L, Sweredoski MJ, Carlisle HJ, Graham RL, Hess S, Kennedy MB: Phosphorylation of synaptic GTPase-activating protein (synGAP) by $\mathrm{Ca}^{2+} /$ calmodulin-dependent protein kinase II (CaMKII) and cyclin-dependent kinase 5 (CDK5) alters the ratio of its GAP activity toward ras and rap GTPases. $J$ Biol Chem 2015, 290:4908-4927.

The authors posit that phosphorylation of synGAP by CaMKII shifts the dendrite toward a greater rate of addition of AMPA receptors, and phosphorylation by Cdk5 shifts the rate back toward greater removal of AMPA receptors.

**37. Walkup WG, Mastro TL, Schenker LT, Vielmetter J, Hu R, Iancu A, Reghunathan M, Bannon BD, Kennedy MB: A model for regulation by SynGAP-alpha1 of binding of synaptic proteins to PDZ-domain 'Slots' in the postsynaptic density. Elife 2016, 5:e16813.

SynGAP acts as a placeholder, binding to PDZ domains on PSD-95 that can also immobilize AMPA receptors. Phosphorylation of synGAP by CaMKII frees PDZ domains, making room for more AMPA receptors.

38. Vazquez LE, Chen HJ, Sokolova I, Knuesel I, Kennedy MB: SynGAP regulates spine formation. $J$ Neurosci 2004, 24:8862-8872.

39. Jackson AC, Nicoll RA: The expanding social network of ionotropic glutamate receptors: TARPs and other transmembrane auxiliary subunits. Neuron 2011, 70:178-199.

40. Siddiqui TJ, Pancaroglu R, Kang Y, Rooyakkers A, Craig AM: LRRTMs and neuroligins bind neurexins with a differential code to cooperate in glutamate synapse development. J Neurosci 2010, 30:7495-7506. 


\section{Figure Legends}

Figure 1. In $\mathrm{PKM} \zeta$ null mutant mice, $\mathrm{PKC} 1 / \lambda$ compensates for the normal function of PKM $\zeta$. A) In Wild-type mice, tetanization increases the amount of PKM $\zeta$ at $3 \mathrm{hrs}$ after stimulus, but does not increase the amount or phosphorylation of $\mathrm{PKC} / \lambda$. In $\mathrm{PKM} \zeta$ null mice, tetanization increases the amount of $\mathrm{PKCl} / \lambda$ and its phosphorylated form. $\mathrm{B}$ ) Maintenance of late phase LTP in hippocampal slices from PKM $\zeta$ null mice (filled circles) is reversed by application of the $\mathrm{PKCl} / \lambda$ antagonist, ICAP $(10 \mu \mathrm{M})$ when it is applied $3 \mathrm{hrs}$ post tetanus. Tetanus was applied at the time indicated by the arrow. The inset shows representative extracellular recorded EPSPs (field Excitatory PostSynaptic Potentials) at the time points indicated by small numbers. C) ICAP has no effect on maintenance of late LTP in wild type hippocampal slices (filled circles). As expected, inhibition of $\mathrm{PKC} / \lambda$ by ICAP blocks induction of LTP in a second pathway in the same wild type slice (open circles). This control shows that the inhibitor is working. The inset on the right is an additional control showing that, in the absence of ICAP, LTP can be induced in a second pathway by tetanization after $270 \mathrm{~min}$ of incubation of the slice. Reproduced from Tsokas et al. eLife 2016;5:e14846. DOI: 10.7554/eLife.14846 [23]

Figure 2. Activation of CaMKII by NMDA receptors causes synGAP $\alpha 1$ and synGAP $\alpha 2$ to move away from the postsynaptic membrane. SynGAP moves into the periphery of the PSD, which the authors refer to as the "pallium." Hippocampal cultures were fixed and stained by immunogold-labeling for the isoform of synGAP that contains a PDZ ligand $(\alpha 1)$ and the isoform that does not $(\alpha 2)$. A) Control labeling; B) Cultures were incubated with tatCN21, an inhibitor of CaMKII; C) Cultures were exposed to $50 \mu \mathrm{M}$ NMDA for 2 min; D) Cultures were treated with NMDA and a control, scrambled peptide that does not inhibit CaMKII (tatCtrl); E) Cultures were treated with NMDA and tatCN21. Reproduced from Yang et al. (2013) PLoS ONE 8(8): e71795. doi:10.1371/journal.pone.0071795 [31].

Figure 3. Altered composition of the postsynaptic density in mice with heterozygous deletion of synGAP. In these experiments, postsynaptic densities were prepared by standard procedures from pooled brains of six synGAP heterozygous (HET) mice and six wild type (WT) litter mates. The ratios of amounts of each protein to the amount of PSD-95 in the two postsynaptic density preparations were determined from quantitative immunoblots scanned with a Li-Cor Odyssey scanner. Individual points (n) are technical replicates of the ratios determined from single lanes on sets of gels each containing six lanes of $5 \mu \mathrm{g}$ wild type PSD and six lanes of $5 \mu \mathrm{g}$ synGAP HET PSD. A) Ratios of synGAP to PSD-95 in WT and HET. $p=0.0007$; Cohen's d (effect size) $=1.75$. B) Ratios of TARPs to PSD-95 in WT and HET. $p=0.017$, Cohen's $d=0.93$. C) Ratios of LRRTM2 to PSD-95 in WT and 
HET. $\mathrm{p}=0.0035$, Cohen's $\mathrm{d}=0.66$. Reproduced from Walkup et al. eLife 2016;5:e16813. DOI: 10.7554/eLife.16813 [37]. 


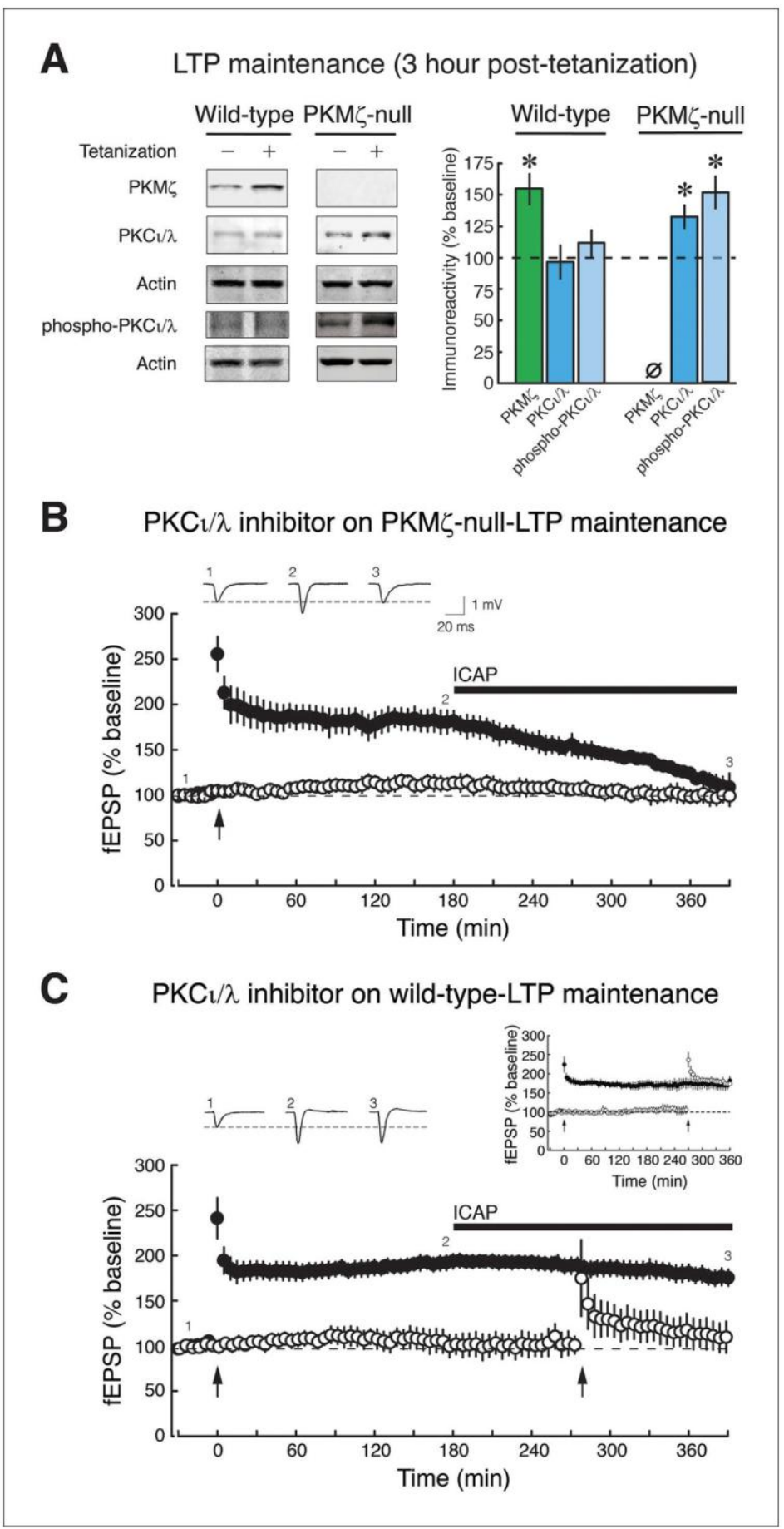




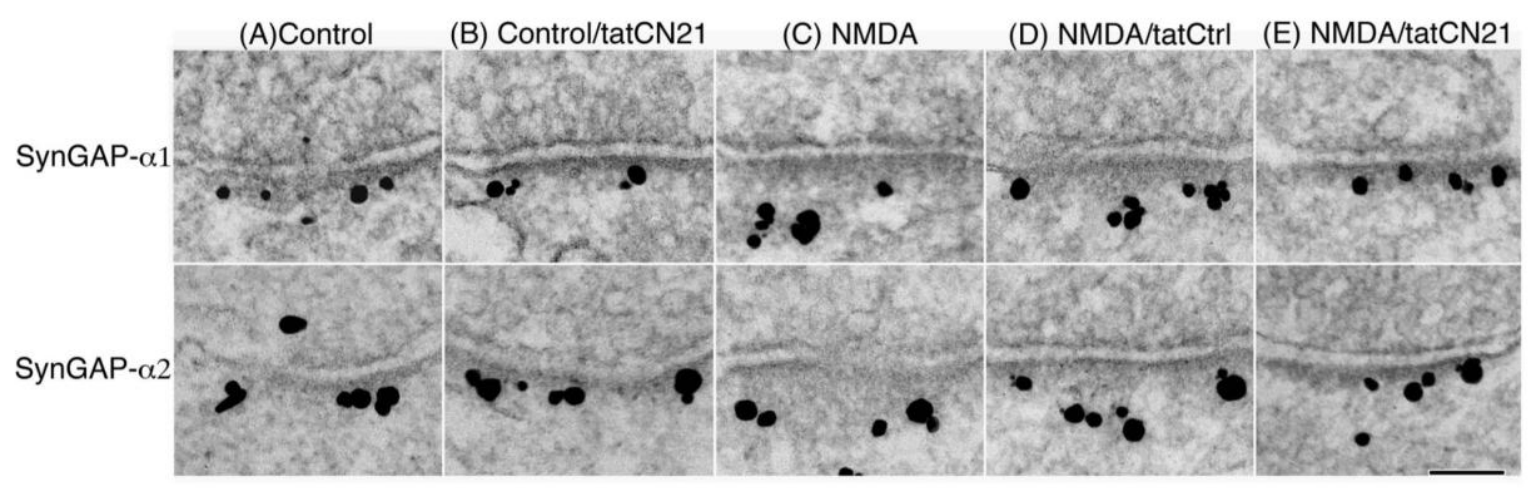



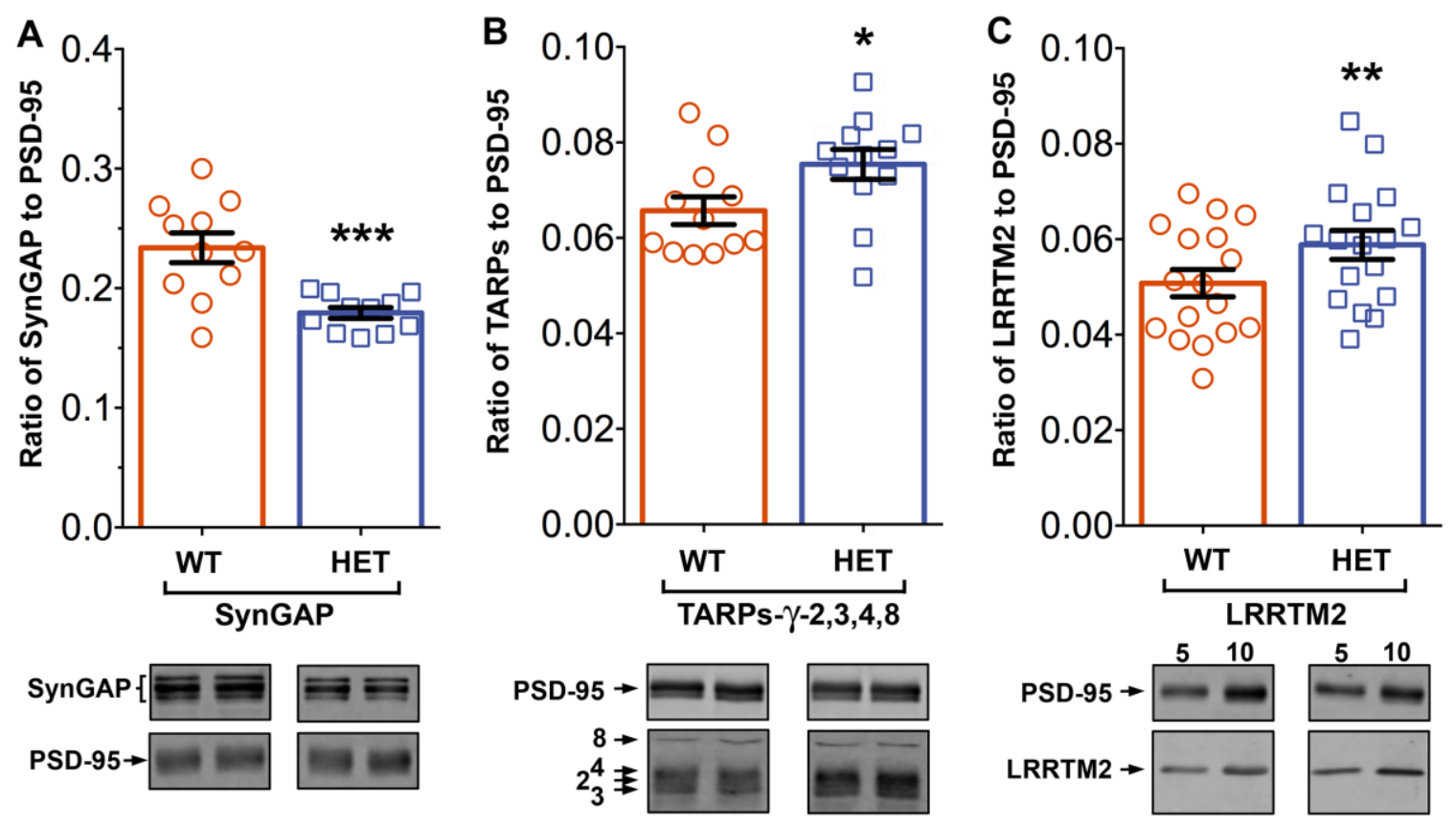